\title{
A CLASS OF ELLIPTIC EQUATIONS RELATED TO OPTICAL DESIGN
}

\author{
J. Rubinstein And G. Wolansky
}

\begin{abstract}
We use a local geometric construction to derive a class of elliptic differential operators. The requirement that the operator will be a derivative of a functional leads to a natural characterization of a family of operators that connect the classical Laplace operator to the mean curvature operator. We further show that the elliptic operators derived here have a canonical interpretation in the framework of optical design.
\end{abstract}

\section{Introduction}

A classical problem in optical design is to find an optical surface that converts a given incident wavefront into a refracted wavefront with desired properties. An optical surface is either a surface separating two media with different refraction indices, or a reflecting surface (mirror). In this paper we shall provide a solution for this problem in the case where the incident wavefront is a parallel surface, and the refracted wavefront is described by its mean curvature at the point of intersection with the refractive surface. Our solution is in the form of a differential equation for the graph of the refractive surface.

We are particularly interested in the case where the refracted wavefront is a minimal surface. In this case the differential equation has a simple variational interpretation. The variational problem is particularly interesting in the case where the refraction index in the domain of the incident wavefront is larger than the refraction index in the domain of the refracted wavefront.

We start in the following section by providing a condition for a general family of differential elliptic operators to be a derivative of a functional. We proceed in section 3 to define a large family of elliptic operators through a local geometric construction. The additional condition, that each operator in that large family is a variational operator, gives rise to a geometric criterion that is similar to Snell's law from geometrical optics. Moreover, this condition restricts the large family to a more specific family of operators that provide a natural link between the Laplace operator and the mean curvature operator.

In section 4 we solve the eikonal equation in a local neighborhood of the refractive surface. We then show that the variational operators derived geometrically in section 3 provide a solution to the optical design problem referred to

Received September 7, 2001.

2000 Mathematics Subject Classification. 35J20, 35J60, 78A05. 
above for the case where the refracted wavefront has zero mean curvature. We further derive an equation for the case where the refracted wavefront has any prescribed mean curvature.

In section 5 we elaborate on the variational interpretation of the optical design problem. In particular we discuss the case of wave propagation from a medium of high refraction index to a medium with low refraction index. We also point out the similarity between the optical design problem and the electrostatic BornInfeld model.

Remark. The case of wave reflection by mirrors can be considered as a special case of refraction. This special case is analyzed towards the end of the paper.

\section{Differential operators}

Let $D \subset \mathbb{R}^{2}$ be a smooth domain. For a given $u \in C^{1}(D)$, define $\partial_{\nabla u}^{2}$ to be the operator of second derivative in the directions of $\nabla u:=\left(u_{x}, u_{y}\right)$, and let $\bar{\partial}_{\nabla u}^{2}$ be the second derivative in the perpendicular direction $\bar{\nabla} u:=\left(-u_{y}, u_{x}\right)$. Let $q_{1}$, $q_{2}$ be a pair of positive valued functions on $\mathbb{R}^{+}$such that

$$
q_{1}(0)=q_{2}(0):=\bar{q} .
$$

Now define the following operator $L$ on $C^{2}(D)$ :

$$
L(u)=q_{1}\left(|\nabla u|^{2}\right) \partial_{\nabla u}^{2}+q_{2}\left(|\nabla u|^{2}\right) \bar{\partial}_{\nabla u}^{2} .
$$

We remark that the derivatives $\partial_{\nabla u}^{2}$ and $\bar{\partial}_{\nabla u}^{2}$ are not defined only if $\nabla u=0$. In that case we use (1) to define $L(u)$ consistently as $\bar{q} \Delta u$.

There are two special cases in which $L$ reduces to well known operators. The first case is $q_{1} \equiv q_{2} \equiv 1$, where $L$ is the Laplacian operator. The second case is $q_{1}(s)=(1+s)^{-3 / 2}, q_{2}(s)=(1+s)^{-1 / 2}$, where $L$ becomes the mean curvature operator of the graph of $u$, which, in a more standard notation, is given by $\nabla \cdot \frac{\nabla u}{\sqrt{1+|\nabla u|^{2}}}$. In these two examples, the operator $L$ is a formal derivative of a variational principle for a functional of the form:

$$
\int_{D} Q\left(|\nabla u|^{2}\right)
$$

where $Q$ is given by $Q(s)=s$ for the Laplacian, and $Q(s)=\sqrt{1+s}$ for the mean curvature operator.

There are further cases where $L$ is associated with a functional of the from (3). They are characterized by the following Lemma.

Lemma 2.1. The operator $L$ is formally derived from a variational principle $\int Q\left(|\nabla u|^{2}\right)$ if and only if

$$
q_{2}+2 s \frac{d q_{2}}{d s}=q_{1}
$$

In this case, $Q^{\prime}=q_{2}$. 
Proof. If $L$ is formally derived from a variational functional of this form, then

$$
\frac{1}{2} L u=\frac{\partial\left(Q^{\prime}\left(|\nabla u|^{2}\right) u_{x}^{\prime}\right)}{\partial x}+\frac{\partial\left(Q^{\prime}\left(|\nabla u|^{2}\right) u_{y}^{\prime}\right)}{\partial y} .
$$

Since the functional is invariant under rotation, we may apply a rotation of the coordinates centered at a given point $(x, y)$ without changing $L u$ at this point. Applying such a rotation for which $u_{y}^{\prime}=0$. implies

$$
u_{x x}^{\prime \prime} \rightarrow \partial_{\nabla u}^{2} u \quad, \quad u_{y y}^{\prime \prime} \rightarrow \bar{\partial}_{\nabla u}^{2} \quad|\nabla u|^{2} \rightarrow\left(u_{x}^{\prime}\right)^{2}
$$

at the given point. In these local coordinates, the operator $L$ takes the form

$$
\frac{1}{2} L u(x, y)=\left[Q^{\prime}\left(\left(u_{x}^{\prime}\right)^{2}\right)+2 Q^{\prime \prime}\left(\left(u_{x}^{\prime}\right)^{2}\right)\left(u_{x}^{\prime}\right)^{2}\right] u_{x x}^{\prime \prime}+\left[Q^{\prime}\left(\left(u_{x}^{\prime}\right)^{2}\right)\right] u_{y y}^{\prime \prime}
$$

Transforming back to the coordinate-independent notation (4) and comparing with (2) we obtain

$$
q_{2}(s)=Q^{\prime}(s) \quad ; \quad q_{1}(s)=Q^{\prime}(s)+2 s Q^{\prime \prime}(s) .
$$

\section{A geometric construction}

We construct a class of operators of the form $L$ through a canonical geometrical procedure. Let $\mathbf{e}_{1}, \mathbf{e}_{2}, \mathbf{e}_{3}$ be the canonical base of coordinates in $\mathbb{R}^{3}$. The graph of a function $u$ on $D$ is given by $x \mathbf{e}_{1}+y \mathbf{e}_{2}+u(x, y) \mathbf{e}_{3}$. The unit normal to this graph above a point $(x, y) \in D$ is given by

$$
\mathbf{N}=\left(1+|\nabla u|^{2}\right)^{-1 / 2}\left(-u_{x}^{\prime},-u_{y}^{\prime}, 1\right)
$$

Consider a point $\left(x_{0}, y_{0}, u\left(x_{0}, y_{0}\right)\right)$ on the graph of $u$, and assume $\left|\nabla u\left(x_{0}, y_{0}\right)\right| \neq$ 0 . Then the plane $P\left(\mathbf{e}_{3}, \mathbf{N}\right)$ spanned by $\mathbf{e}_{3}$ and $\mathbf{N}$ is defined at this point.

Let $\hat{\mathbf{e}}_{3}$ to be a unit vector in $P\left(\mathbf{e}_{3}, \mathbf{N}\right)$, obtained by a rotation of $\mathbf{N}$ by an angle $\theta$ towards $\mathbf{e}_{3}$. Note that the angle $\gamma$ between $\mathbf{e}_{3}$ and $\mathbf{N}$ is given by

$$
\cos \gamma=\mathbf{N} \cdot \mathbf{e}_{3}=\frac{1}{\sqrt{1+|\nabla u|^{2}}}
$$

We complete $\hat{\mathbf{e}}_{3}$ to a normal triplet $\left\{\hat{\mathbf{e}}_{1}, \hat{\mathbf{e}}_{2}, \hat{\mathbf{e}}_{3}\right\}$ centered at the point $\left(x_{0}, y_{0}\right.$, $\left.u\left(x_{0}, y_{0}\right)\right)$. Let $\hat{u}$ be the function whose graph $\hat{x} \hat{\mathbf{e}}_{1}+\hat{y} \hat{\mathbf{e}}_{2}+\hat{u}(\hat{x}, \hat{y}) \hat{\mathbf{e}}_{3}$ coincides with the graph of $u$ in the neighborhood of $\left(x_{0}, y_{0}, u\left(x_{0}, y_{0}\right)\right)$. Define

$$
\Delta_{\theta} u\left(x_{0}, y_{0}\right)=\left.\frac{\partial^{2} \hat{u}}{\partial^{2} \hat{x}}\right|_{(0,0)}+\left.\frac{\partial^{2} \hat{u}}{\partial^{2} \hat{y}}\right|_{(0,0)} \equiv \hat{\Delta} \hat{u}(0,0) \text { if }\left|\nabla u\left(x_{0}, y_{0}\right)\right| \neq 0 .
$$

Notice that $\theta$ is still unspecified at this stage. We shall later specialize to the case where $\theta$ depends on $|\nabla u|$. However, we set, at this stage, $\theta=\gamma$ if $|\nabla u|=0$, so that $\Delta_{\theta} u$ coincides with the usual Laplacian at critical points of $u$, namely

$$
\Delta_{\gamma} u\left(x_{0}, y_{0}\right)=\Delta u\left(x_{0}, y_{0}\right) \quad \text { if }\left|\nabla u\left(x_{0}, y_{0}\right)\right|=0 .
$$


Remark. The above definition is well posed for any twice differentiable $u$. In particular, it is independent of the choice of the pair $\left(\hat{\mathbf{e}}_{1}, \hat{\mathbf{e}}_{2}\right)$, as long as $\left\{\hat{\mathbf{e}}_{1}, \hat{\mathbf{e}}_{2}, \hat{\mathbf{e}}_{3}\right\}$ makes an orthogonal triplet.

We obtain, in this way, a family of operators $\Delta_{\theta}$. Evidently, $\Delta_{\gamma} \equiv \Delta$ - the classical Laplacian. It can also be seen that $\Delta_{0}$ is the mean curvature operator. Indeed, if $\theta=0$ then $\hat{\mathbf{e}}_{3}$ coincides with the local normal $\mathbf{N}$ and $(0,0)$ is thus a critical point of $\hat{u}$. Since the mean curvature of a graph of a function is identical to half its Laplacian at a critical point, on the one hand, and is invariant to rotation of the graph on the other, our claim follows.

To obtain an explicit expression for $\Delta_{\theta}$, assume that the graph is rotated at the $(x, y)$ plane (leaving $\mathbf{e}_{3}$ unchanged), so that $u_{y}^{\prime}=0$ at $\left(x_{0}, y_{0}\right)$. We may also assume that $x_{0}=y_{0}=u\left(x_{0}, y_{0}\right)=0$. Then

$$
u(x, y)=x u_{x}^{\prime}+\frac{1}{2}\left(x^{2} u_{x}^{\prime \prime}+2 x y u_{x, y}^{\prime \prime}+y^{2} u_{y}^{\prime \prime}\right)+O\left(x^{3}+y^{3}\right)
$$

Let us now rotate $\mathbf{e}_{3}$ by an angle $\theta+\gamma$ without changing $\mathbf{e}_{2}$, i.e. we take $\hat{\mathbf{e}}_{2}=\mathbf{e}_{2}$. In that case

$$
\begin{gathered}
\hat{x}=x \cos (\theta+\gamma)+u \sin (\theta+\gamma), \\
\hat{y}=y, \\
\hat{u}=-x \sin (\theta+\gamma)+u \cos (\theta+\gamma) .
\end{gathered}
$$

Inverting these equations and using (5), we obtain an expansion for $x(\hat{x}, \hat{y})$ :

$$
\begin{gathered}
x=\frac{\hat{x}}{\cos (\theta+\gamma)+u_{x}^{\prime} \sin (\theta+\gamma)} \\
-\frac{\sin (\theta+\gamma)}{\cos (\theta+\gamma)+u_{x}^{\prime} \sin (\theta+\gamma)}\left[\frac{u_{x x}^{\prime \prime} \hat{x}^{2}}{2\left(\cos (\theta+\gamma)+u_{x}^{\prime} \sin (\theta+\gamma)\right)^{2}}\right. \\
\left.+\frac{u_{x y}^{\prime \prime} \hat{x} \hat{y}}{\cos (\theta+\gamma)+u_{x}^{\prime} \sin (\theta+\gamma)}+\frac{u_{y y}^{\prime \prime} \hat{y}^{2}}{2}\right]+O\left(\hat{x}^{3}+\hat{y}^{3}\right) .
\end{gathered}
$$

Substituting (7) in the third equation of (6), and using (5) we obtain

$$
\Delta_{\theta} \hat{u}(0,0)=\frac{u_{x x}^{\prime \prime}}{\left(\cos (\theta+\gamma)+u_{x}^{\prime} \sin (\theta+\gamma)\right)^{3}}+\frac{u_{y y}^{\prime \prime}}{\cos (\theta+\gamma)+u_{x}^{\prime} \sin (\theta+\gamma)}
$$

We now use the formula $\sin \gamma=u_{x}^{\prime} / \sqrt{1+\left(u_{x}^{\prime}\right)^{2}}$ and some trigonometric identities to obtain

$$
\cos (\theta+\gamma)+u_{x}^{\prime} \sin (\theta+\gamma)=\frac{\cos \theta}{\cos \gamma}
$$

Finally we rotate back to the original $x$ and $y$ variables by removing the restriction $u_{y}^{\prime}=0$. This amounts to replacing $u_{x}^{\prime}$ by $|\nabla u|, u_{x x}^{\prime \prime}$ by $\partial_{\nabla u}^{2}$ and $u_{y y}^{\prime \prime}$ by $\bar{\partial}_{\nabla u}^{2}$. As a result we obtain

$$
\Delta_{\theta} u=q^{3}\left(|\nabla u|^{2}\right) \partial_{\nabla u}^{2}+q\left(|\nabla u|^{2}\right) \bar{\partial}_{\nabla u}^{2}
$$


where

$$
q(s)=\frac{1}{\cos \theta \sqrt{1+s}}
$$

Lemma 3.1. The operator $\Delta_{\theta}$ is a formal derivative of a functional of the form $\int Q\left(|\nabla u|^{2}\right)$ if and only if there exists a constant $\mu \in \mathbb{R}$ for which

$$
\sin \theta=\mu \sin \gamma
$$

i.e

$$
\theta(|\nabla u|)=\arcsin \left[\mu \frac{|\nabla u|}{\sqrt{1+|\nabla u|^{2}}}\right] .
$$

Proof. By Lemma 2.1, the condition for the existence of a variational principle for $\Delta_{\theta}$ is that $q$ will be a solution of

$$
q+2 s \frac{d q}{d s}=q^{3} .
$$

However, all solutions of this equation are of the form $q(s)=(1+\alpha s)^{-1 / 2}$, where $\alpha \in \mathbb{R}$. Using (10) we obtain

$$
\cos \theta(s)=\sqrt{\frac{1+\alpha s}{1+s}} \rightarrow \sin \theta=\mu \sin \gamma
$$

where $\mu= \pm \sqrt{1-\alpha}$.

\section{Optical design}

The condition (11) derived in Lemma 3.1 has an optical interpretation. Consider a ray in the direction of the original $\mathbf{e}_{3}$ direction propagating in a medium with a refraction index $n_{1}$ towards the surface $u$. Assume further that the refraction index in the medium above $u$ is $n_{2}$, and denote the ratio between the refraction indices by $\mu=n_{1} / n_{2}$. Then, since (11) can be identified with Snell's law of geometrical optics, the ray will be refracted by the surface $u$ into a new ray in the direction of $\hat{\mathbf{e}}_{3}$. This observation motivates us to consider whether the differential operator $\Delta_{\theta}$ has an optical meaning.

To answer this question, let us indeed consider the surface $u$ as a refractive optical element. Specifically, we assume that the subgraph $\{z<u(x, y)\}$ is occupied by a medium with a refraction index $n_{1}$, while in the supergraph, $\{z>u(x, y)\}$ the refraction index is $n_{2}$. Consider an incoming planar wavefront orthogonal to the $z$ axis, i.e. a parallel beam of rays in the direction of the positive $z$ axis. The rays are refracted at the interface $u$ according to Snell's law. Therefore the wavefront will be distorted. The local optical power of $u$ is determined by the mean curvature of the new front at each point. The curvature of the distorted wavefront was calculated by Keller and Keller [3] and by Kneisly [4]. We present a different calculation method that leads to an explicit differential equation for the refractive surface. 
A common goal in optics is to design an optical element with a prescribed optical power. Therefore we want to find $u$ such that the mean curvature of the refracted wavefront at its intersection point with $u$ is a prescribed function $H(x, y)$. In addition we may require $u$ to satisfy certain conditions on the boundary of a domain $D \subset \mathbb{R}^{2}$.

To derive an equation for $u$ we recall from the theory of geometrical optics that the wavefronts are the level surfaces of the eikonal function $\mathcal{S}$. The eikonal satisfies the equation [1]

$$
|\nabla \mathcal{S}|=n(x, y, z)
$$

where $n(x, y, z)=n_{1}$ if $z<u(x, y)$ and $n(x, y, z)=n_{2}$ if $z>u(x, y)$. Since the beam is parallel to the $(x, y)$ plane, we may set $\mathcal{S}\left(x, y, z_{0}\right) \equiv 0,(x, y) \in D$, on the plane $z=z_{0}<u$. For simplicity we set $z_{0}=0$. Hence, $\mathcal{S}(x, y, u(x, y))=n_{1} u(x, y)$ on $D$. The solution of the eikonal in the domain $z>u$ is determined by the hyperbolic problem

$$
|\nabla \mathcal{S}|=n_{2}, \quad \mathcal{S}(x, y, u(x, y))=n_{1} u(x, y),
$$

in the supergraph domain. Since the optical power of the lens is given by the mean curvature of the eikonal surface at the interface, we formulate the following problem:

P: Find a function $u$ defined on a planner domain $D$ for which the level surfaces of the solution $S$ of (12) have a prescribed mean curvature at each point $(x, y, u(x, y))$.

To solve this problem we define a coordinate triplet $\left\{\hat{\mathbf{e}}_{1}, \hat{\mathbf{e}}_{2}, \hat{\mathbf{e}}_{3}\right\}$ for each point on the graph, where $\hat{\mathbf{e}}_{3}$ is directed along the outgoing ray at this point. Then, we need to compute the Laplacian of $\mathcal{S}$ at this point with respect to these coordinates. Moreover, we shall express this Laplacian in terms of the refractive surface $u$. For this purpose we need to compute, up to second order, the value of $\mathcal{S}$ on the tangent plane to the wavefront at this point. Since this plane is perpendicular to $\hat{\mathbf{e}}_{3}$ by definition, we can do it by a direct geometrical consideration:

Let $(\hat{x}, \hat{y}, \hat{z})$ be the coordinates of a point in the basis $\left\{\hat{\mathbf{e}}_{1}, \hat{\mathbf{e}}_{2}, \hat{\mathbf{e}}_{3}\right\}$. The origin of this system is at some point $P_{0}=\left(x_{0}, y_{0}, u\left(x_{0}, y_{0}\right)\right)$ on the graph (see Figure 1). A point $P_{1}$ on the graph in a neighborhood of $P_{0}$ is represented in the new coordinates by $\vec{r} \equiv \hat{x} \hat{\mathbf{e}}_{1}+\hat{y} \hat{\mathbf{e}}_{2}+\hat{u} \hat{\mathbf{e}}_{3}$. We have

$$
S\left(P_{1}\right)-S\left(P_{0}\right)=n_{1} \vec{r} \cdot \mathbf{e}_{3}
$$

where $\mathbf{e}_{3}$ is the direction of the incoming ray.

Let $P$ be an arbitrary point in the neighborhood of $P_{0}$. We express the eikonal at $P$ by expanding it in a Taylor series in the $\hat{z}$ variable:

$$
S(P)=S\left(P_{0}\right)+S_{0}(\hat{x}, \hat{y})+\hat{z}\left(n_{2}+S_{1}(\hat{x}, \hat{y})\right)+\frac{\hat{z}^{2}}{2} S_{2}(\hat{x}, \hat{y})+\frac{\hat{z}^{3}}{6} S_{3}(\hat{x}, \hat{y}, \hat{z}) .
$$




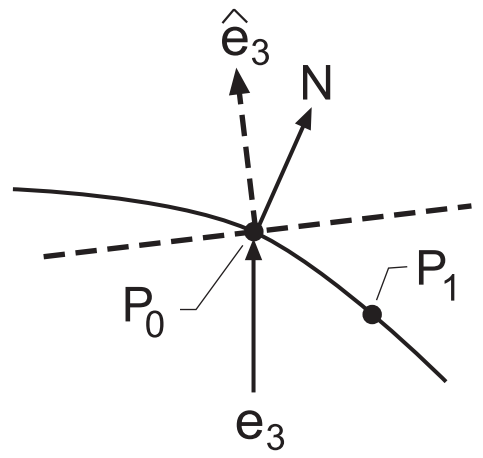

FiguRE 1. The coordinate systems used in the geometric construction

Substituting the expansion (14) in equation (12) we obtain (to second order in $\hat{z})$ :

$$
\begin{aligned}
\left|n_{2}+S_{1}+\hat{z} S_{2}+\frac{\hat{z}^{2}}{2} S_{3}\right|^{2}+\mid \partial_{\hat{x}} S_{0}+ & \hat{z} \partial_{\hat{x}} S_{1}+\left.\frac{\hat{z}^{2}}{2} \partial_{\hat{x}} S_{2}\right|^{2} \\
& +\left|\partial_{\hat{y}} S_{0}+\hat{z} \partial_{\hat{y}} S_{1}+\frac{\hat{z}^{2}}{2} \partial_{\hat{y}} S_{2}\right|^{2}=n_{2}^{2} .
\end{aligned}
$$

Expanding in $\hat{z}$ we obtain to leading order

$$
\left(n_{2}+S_{1}\right)^{2}+\left|\partial_{\hat{x}} S_{0}\right|^{2}+\left|\partial_{\hat{y}} S_{0}\right|^{2}=n_{2}^{2} .
$$

The choice of the coordinates system implies at once

$$
\partial_{\hat{x}} S_{0}(0,0)=\partial_{\hat{y}} S_{0}(0,0)=0 .
$$

Therefore it follows from (16) that

$$
S_{1}(0,0)=0 .
$$

Moreover, differentiating (16) with respect to $\hat{x}$ and $\hat{y}$, we obtain also

$$
\partial_{\hat{x}} S_{1}(0,0)=\partial_{\hat{y}} S_{1}(0,0)=0 .
$$

Proceeding to the linear (in $\hat{z}$ ) terms in (15), we similarly find

$$
S_{2}(0,0)=0 \text {. }
$$

We now choose $P=P_{1}$ in (14), and use (13) to write the the eikonal on the plane tangent to the wavefront at $P_{0}$ in terms of $\hat{u}$ (up to second order in $\hat{u}$ ):

$$
S_{0}(\hat{x}, \hat{y})=n_{1} \vec{r} \cdot \overrightarrow{\mathbf{e}}_{3}-n_{2} \hat{u}-\hat{u} S_{1}-\frac{\hat{u}^{2}}{2} S_{2},
$$

i.e.

$$
\begin{array}{r}
S_{0}(\hat{x}, \hat{y})=\left(n_{1} \hat{\mathbf{e}}_{3} \cdot \mathbf{e}_{3}-n_{2}\right) \hat{u}(\hat{x}, \hat{y})+n_{1}\left(\hat{x} \hat{\mathbf{e}}_{1} \cdot \mathbf{e}_{3}+\hat{y} \hat{\mathbf{e}}_{2} \cdot \mathbf{e}_{3}\right)- \\
\hat{u}(\hat{x}, \hat{y}) S_{1}(\hat{x}, \hat{y})-\frac{\hat{u}^{2}}{2} S_{2}(\hat{x}, \hat{y}) .
\end{array}
$$


The mean curvature of the level surface of $S$ at the point $P_{0}$ is given by half the Laplacian of $S_{0}$ divided by $n_{2}$ at the point $\hat{x}=\hat{y}=0$. From the formula we derived for $S_{1}$ and its gradient and for $S_{2}$ at $(0,0)$, and since $\hat{u}(0,0)=0$, then twice the required optical power is given by the following operator:

$$
\mathcal{D}_{n_{1}, n_{2}} u(x, y)=\frac{1}{n_{2}}\left(n_{1} \hat{\mathbf{e}}_{3} \cdot \mathbf{e}_{3}-n_{2}\right) \Delta \hat{u}(\hat{x}, \hat{y}) .
$$

Using $(9,10)$ we return to the original coordinates and write:

$$
\mathcal{D}_{n_{1}, n_{2}} u=\frac{1}{n_{2}}\left(n_{1} \cos \theta-n_{2}\right) \Delta_{\theta} u
$$

where $\theta$ is the angle between $\mathbf{e}_{3}$ and $\hat{\mathbf{e}}_{3}$, i.e. the angle between an incoming ray and its refracted outgoing ray. To write the operator explicitly in terms of the $(x, y)$ coordinates, we use Snell's law. It is convenient to recall the parameter $\mu$ defined above, and to introduce the additional notation $\beta=1-\mu^{2}$. The angle $\psi$ between the incoming ray $\mathbf{e}_{3}$ and the normal to surface $N$ is

$$
\sin \psi=\frac{|\nabla u|}{\sqrt{1+|\nabla u|^{2}}}
$$

while the angle $\psi^{\prime}$ between $N$ and the outgoing ray $\hat{\mathbf{e}}_{3}$ satisfies

$$
\sin \psi^{\prime}=\mu \sin \psi
$$

The angle $\theta$ between $\mathbf{e}_{3}$ and $\hat{\mathbf{e}}_{3}$ is $\theta=\psi-\psi^{\prime}$. A direct computation yields

$$
\cos \theta+|\nabla u| \sin \theta=\sqrt{1+\beta|\nabla u|^{2}},
$$

while

$$
\hat{\mathbf{e}}_{3} \cdot \hat{\mathbf{e}}=\cos \left(\psi-\psi^{\prime}\right)=\frac{\sqrt{1+\beta|\nabla u|^{2}}+\mu|\nabla u|^{2}}{1+|\nabla u|^{2}} .
$$

We substitute (20) in (8) to obtain an expression for the optical power operator $\mathcal{D}_{n_{1}, n_{2}} u$ in the $(x, y)$ variables. We obtain

$$
\mathcal{D}_{n_{1}, n_{2}} u=p\left(|\nabla u|^{2}\right)\left[\left(1+\beta|\nabla u|^{2}\right)^{-3 / 2} \partial_{\nabla u}^{2}+\left(1+\beta|\nabla u|^{2}\right)^{-1 / 2} \bar{\partial}_{\nabla u}^{2}\right],
$$

where

$$
p(s)=\mu \frac{\sqrt{1+\beta s}+\mu s}{1+s}-1 .
$$

Problem $\mathbf{P}$ can now be written in the form $\mathcal{D}_{n_{1}, n_{2}} u=2 H(x, y)$. Using the proof of Lemma 3.1, we can write a divergence form for the homogeneous version of $\mathbf{P}$.

Lemma 4.1. Any solution of the equation

$$
\nabla \cdot \frac{\nabla u}{\sqrt{1+\beta|\nabla u|^{2}}}=0
$$

represents a solution of problem $\mathbf{P}$ with mean curvature 0. 
Remark. It is useful to notice at this point that the case in which the surface $u$ is a mirror, reflecting the incoming parallel wavefront, can be considered as a special case of refraction. all we have to do is to set $n_{2}=-n_{1}$ in the preceding analysis. Note that this implies an appropriate change of sign in some of the formulas.

\section{The variational principle}

We want to analyze the boundary value problem consisting of (23) and the boundary condition

$$
u=g, \quad(x, y) \in \partial D,
$$

where $g$ a smooth (Lipschitz) function. The homogeneous equation (23) can be derived from a variational principle. Consider the functional

$$
F_{\beta}(u)=\int_{D} \frac{1}{\beta} \sqrt{1+\beta|\nabla u|^{2}} .
$$

Clearly the operator on the left hand side of (23) is the first derivative of $F_{\beta}$. We expect the solutions to (23) to be minimizers of (25) subject to the boundary constraint (24).

The functional (25) has two interpretations besides the optical and geometrical problems we mentioned so far ${ }^{1}$. Both interpretations are for negative values of $\beta$. The first example is in the special case $\beta=-1$. The variational problem is now to maximize the functional $\int_{D} \sqrt{1-|\nabla u|^{2}}$, i.e. to find a maximal space-like surface in the Minkowsky metric. This problem was considered over $\mathbb{R}^{n}$ in [2]. The second example is the electrostatic Born-Infeld model. This problem was considered, e.g. by Yang [5], again for the case of the entire space.

To proceed with the boundary value problem we shall consider separately three cases. We first consider refraction of a wavefront propagating from one medium into another medium with a higher refraction index. In this case we have

Theorem 5.1. Assume $n_{1}<n_{2}$. Given a bounded Lipschitz domain $D \subset \mathbb{R}^{2}$ and a boundary condition (24), there exists a smooth solution for (23), subject to the boundary condition. The solution is a minimizer (subject to a similar boundary constraint) of the functional $F_{\beta}$.

The proof is straightforward, since under the Theorem's assumptions $\beta$ is positive. The functional $F_{\beta}$ is convex (which implies existence of minimizers), and the elliptic equation guarantees smoothness for the solution.

The second special case is when $u$ is a mirror surface. As mentioned earlier, this is equivalent to setting $n_{2}=-n_{1}$, i.e. $\beta=0$. The functional $F_{\beta}$ then collapses into the Dirichlet integral, and the operator (23) becomes the Laplace operator. We thus obtain the following theorem

\footnotetext{
${ }^{1}$ We thank Y. Yang for bringing references [2] and [5] to our attention
} 
Theorem 5.2. A mirror reflects a parallel wavefront into a wavefront whose mean curvature is zero if and only if the mirror surface is a harmonic function. More generally, a mirror reflects a parallel wavefront into a wavefront whose mean curvature at its intersection points with the mirror is given by $H(x, y)$ if and only if the mirror surface $u$ satisfies

$$
\Delta u=2 H\left(1+|\nabla u|^{2}\right) .
$$

Finally we consider the difficult case in which $n_{1}>n_{2}$. Since now $\mu>1$, it follows that $\beta<0$, and there may be no solutions to equation (23). For example, one might prescribe on $\partial D$ boundary data $g$, such that each smooth extension of it into $D$ satisfies $|\nabla u| \geq-1 / \beta$ on some set in $D$. The physical interpretation of such boundary data is that every extension of it into $D$ has, on some set in $D$, sufficiently large gradient to enforce total reflection. Therefore, a necessary condition for the existence of a solution to (23) is that the boundary data $g$ satisfies a condition of the following form.

The SG condition: The boundary data $g$ will be said to satisfy the small gradient (SG) condition if there exists a smooth extension $u_{0}$ of $g$ into $D$, such that $\left|\nabla u_{0}\right|<-1 / \beta$ everywhere in $D$.

We conjecture that this condition is also sufficient.

Conjecture 5.3. Assume $n_{1}>n_{2}$. Given a bounded Lipschitz domain $D \subset \mathbb{R}^{2}$ and a boundary condition of the form (24), a smooth solution of (23), subject to the boundary condition, exists if and only if $g$ satisfies the $S G$ condition.

The conjecture can be easily verified for simple special cases such as onedimensional or radial setups. To study it for general domains, we want to use the variational formulation. To emphasize that we only deal with 'space-like' surfaces, we define $F_{\beta}$ to be $\infty$ if $|\nabla u|>-1 / \beta$ over a set of positive measure. Clearly there exists a minimizer for $F_{\beta}$ even for boundary constraint $g$ that do not satisfy the SG condition. But in this case the minimizer will hit the obstacle $|\nabla u|=-1 / \beta$ on some set in $D$. When the SG condition holds, the following estimate can be obtained

Proposition 5.4. Assume $n_{1}>n_{2}$. Let $\bar{u}$ be a minimizer of $F_{\beta}$ subject to (24). If $g$ satisfies the $S G$ condition, then

$$
\int_{D} \frac{1}{\sqrt{1+\beta|\nabla \bar{u}|^{2}}}<\infty
$$

Proof. We set for simplicity, and without loss of generality $G(u)=-F_{-1}(u)=$ $\int_{D} \sqrt{1-|\nabla u|^{2}}$. The variational problem is, therefore, to maximize $G$ under the constraint (24). Consider the functional

$$
H(v,|\nabla u|)=\int v+\frac{1}{4 v}-v|\nabla u|^{2} .
$$


One can readily verify that

$$
\inf _{v \geq 0} H(v,|\nabla u|)=G(u),
$$

implying that $H$ is the Legendre transform of $G$. The infimum is obtained at $\bar{v}=\frac{1}{2 \sqrt{1-|\nabla u|^{2}}}$. Moreover,

$$
\sup _{u} \inf _{v} H=\inf _{v} \sup _{u} H \leq C_{2} .
$$

Since $g$ satisfies the SG condition, there exists a function $u_{0}$ such that $\left.u_{0}\right|_{\partial D}=g$, and $\left|\nabla u_{0}\right|<C_{1}<1$ in $D$. Therefore

$$
\int_{D} \bar{v}+\frac{1}{4 \bar{v}}-\bar{v}|\nabla u|^{2} \geq \int_{D} \bar{v}+\frac{1}{4 \bar{v}}-C_{1}^{2} \bar{v}=\int_{D}\left(1-C_{1}^{2}\right) \bar{v}+\frac{1}{4 \bar{v}} .
$$

Hence $\int_{D} \bar{v}<C_{3}$.

We therefore rephrase Conjecture 5.3 as follows:

Conjecture 5.5. Assume $n_{1}>n_{2}$. The functional $F_{\beta}$, subject to boundary condition (24), is minimized at a function $u$ that is space-like, in the sense that $|\nabla u|<-1 / \beta$ in $D$, if and only if $g$ satisfies the $S G$ condition.

\section{References}

[1] M. Born, E. Wolf, Principles of Optics: Electromagnetic theory of propagation, interference and diffraction of light, Pergamon Press, 1980.

[2] S.-Y. Cheng, S.-T. Yau, Maximal space-like hypersurfaces in the Lorenz-Minkowsky spaces, Ann. of Math. (2) 104 (1976), 407-419.

[3] J.B. Keller, H. B. Keller, Determination of reflected and transmitted fields by geometrical optics, J. Opt. Soc. Amer. 40 (1950), 48-52.

[4] J. A. Kneisly, Local curvature of a wavefront in an optical system, J. Opt. Soc. Amer. 54 (1964), 229-235.

[5] Y. Yang, Classical solutions in the Born-Infeld theory, R. Soc. Lond. Proc. Ser. A Math. Phys. Eng. Sci. 456 (2000), 615-640.

Department of Mathematics, Indiana University, Bloomington, IN 47401, U.S.A.

Department of Mathematics, Technion, Haifa 32000, Israel.

E-mail address: jrubinst@indiana.edu

Department of Mathematics, Technion, Haifa 32000, Israel.

E-mail address: gershonw@math.technion.ac.il 